\title{
Aceptabilidad de un snack de nopal deshidratado previamente encurtido
}

\author{
Juan José Aguilar Lugo Marino¹, Eva Rossina Aguilar Lugo Gerez² y Sixto Josué Pérez \\ Campos $^{3}$
}

${ }^{1}$ Universidad Autónoma del Estado de Hidalgo, juanjoseaguilarlugo@yahoo.com.mx, Carr. México-Laredo

Km. 120.5, Actopan, Hgo., Tel.771 7172000 Ext.5401.

${ }^{2}$ Centro Europeo de Postgrado, evarossina66@hotmail.com, Av. Faro No. 2350, Guadalajara, Jal., Tel. 333

1113352.

${ }^{3}$ Universidad Autónoma del Estado de Hidalgo, sixtojosue@gmail.com, Av. Universitaria Km. 1, Tulancingo, Hgo., Tel.771 7172000 Ext. 2460.

\author{
Información del artículo revisado por pares \\ Fecha de aceptación: junio-2021 \\ Fecha de publicación en línea: diciembre-2021 \\ DOI: https://doi.org/10.29105/vtga7.1-129
}

\section{Resumen}

La deforestación por diversas causas está dañando seriamente al planeta y una de sus consecuencias es que muchas zonas afectadas no se vuelven a reforestar por el costo y el trabajo que implica. Las plantaciones, sobre todo maderables, tardan en poderse explotar, por lo que este trabajo propone fomentar el cultivo del nopal para reforestar ya que sus raíces llegan a medir hasta diez metros y esto evita la pérdida de la capa cultivable del suelo, es de fácil cultivo, resistente a las altas temperaturas y falta de agua, por lo que esta propuesta se basa en el desarrollo de un producto basado en esta planta lo que puede apoyar a los productores al tener una venta regular, además al poderlo adquirir directamente del agricultor al desarrollador del producto se mejoran sus ingresos. Algunas propiedades del nopal como por ejemplo la fibra soluble e insoluble lo convierten en un auxiliar en el tratamiento de la diabetes y el producto desarrollado va dirigido a adolescentes y jóvenes, de los cuales tres de cada diez no consumen verduras y uno de cada tres tiene problemas de sobrepeso u obesidad. El producto desarrollado adquiere un sabor ácido y picoso por medio de chile y vinagre (encurtido), se corta en tiras y posteriormente se deshidrata para su conservación. Su presentación es de un snack saludable. Es un estudio del tipo correlacional incluyendo elementos descriptivos. En un panel de degustación usando la Escala de Likert (1 a 5) tuvo buena aceptación en sabor (3.67), pero respecto a su apariencia su valor fue bajo (2.17), por lo que se pretende utilizar otra técnica de deshidratado o combinarla con otras frutas deshidratadas, pero sí fomentar su consumo por su alto valor nutricional y por ser un cultivo que mejora el medioambiente. Por tal motivo el objetivo de esta investigación es conocer el grado de aceptación de un snack saludable que no únicamente beneficie a los consumidores sino al medioambiente y a los productores del campo.

Palabras clave: Snack de nopal, encurtido-deshidratado, deforestación, diabetes, sobrepeso.

\section{Abstract}

Deforestation for various reasons is seriously damaging the planet and one of its consequences is that many affected areas are not reforested again because of the cost and work involved. The plantations, especially timber plantations, take time to be exploited, so this work proposes to promote the cultivation of nopal for reforestation because its roots reach up to ten meters and this prevents the loss of the arable layer of soil, it is easy to grow, resistant to high temperatures and lack of water, so this proposal is based on the development of a product based on this plant which can support producers to have a regular sale, also to be able to acquire it directly from the farmer to the developer of the product improves their income. Some properties of nopal, such as soluble and insoluble fiber, make it aid in the treatment of diabetes, and the product developed is aimed at adolescents and young people, three out of ten of whom do not eat vegetables and one out of three of whom are overweight or obese. The developed product acquires an acid and spicy flavor through chili and vinegar (pickled), is cut into strips, and then dehydrated for preservation. It is presented as a healthy snack. It is a correlational study including descriptive elements. In a tasting panel using the Likert Scale (1 to 5) it had good acceptance in flavor (3.67), but for its appearance, its value was low (2.17), so it is intended to use another dehydration technique or combine it with other dehydrated fruits, but to promote its consumption for its high nutritional value and for being a crop that improves the environment. For this reason, this research aims to determine the degree of acceptance of a healthy snack that benefits consumers and the environment, and farmers.

Keywords: Nopal snack, pickling-dehydration, deforestation, diabetes, overweight.

JEL: M13, O13, Q13. 


\section{INTRODUCCIÓN}

El binomio emprendimiento-innovación tiene más oportunidades para alcanzar el éxito, que el emprendimiento por sí sólo, por lo que se buscó desarrollar un producto con estas dos cualidades y una tercera más: el que generara un beneficio social, en este caso ligado con el medio ambiente, empleabilidad de productores del campo y coadyuvar a los problemas de sobrepeso y obesidad.

Algo que nos preocupa y nos debe ocupar es el deterioro tan fuerte que está sufriendo nuestro planeta, entre la contaminación en todos sus sentidos y la deforestación tan grande, lo cual puede desencadenar daños irreversibles como el calentamiento global que ya estamos padeciendo, cada año son más las hectáreas de bosque que se ven dañadas y no son rehabilitadas, los daños son tanto voluntarios como involuntarios, pero causa a la larga que ese suelo se vea erosionado y ya no sea apto para los cultivos al perder la capa de tierra donde se pueda sembrar algún tipo de especie vegetal.

Un cultivo que puede coadyuvar a que esa capa de tierra no se pierda es el nopal, una especie que tiene mucha resistencia, es decir, tolera altas temperaturas y la falta de agua. $\mathrm{Su}$ cultivo es sumamente sencillo y la producción de pencas (sus hojas) es muy favorable. Otra ventaja que tiene es que sus raíces retienen la parte de tierra cultivable.

Se pretende en esta propuesta que la producción de pencas jóvenes (tiernas) así como la tuna que es la fruta de esta planta, sea para consumo humano que es parte del proyecto y se dejen las pencas de mayor tamaño para que sirvan de alimento animal.

A las pencas jóvenes se les da un tratamiento similar a los encurtidos, es decir, crear una solución de vinagre con agua y chiles jalapeños dejándolas macerar durante tres días para que estas pencas tomen el sabor ligeramente ácido del vinagre, así como un pequeño picor que ofrecen los chiles en el vinagre.

Un último tratamiento que se le proporciona al nopal para conservarlo es la deshidratación, es decir, extraerle el agua suficiente para que el producto no sufra de una descomposición. Con lo que respecta a la presentación final de los nopales es cortado en tiras y colocados en bolsas para venderse como un snack saludable por las propiedades con que cuenta.

El nopal tiene propiedades como su fibra (soluble e insoluble) que es muy útil para el organismo y auxiliar en problemas de salud como es la diabetes.

La parte medular de este trabajo consiste en saber si a los futuros consumidores les resulta atractivo el adquirirlo. Por sus características va orientado a los adolescentes y jóvenes que desean consumir un producto que les resulte agradable a la vista, de buen sabor y que sea de fácil adquisición y consumo. Incluyendo el plus de ser un alimento nutritivo. Por tal motivo se desarrolló una degustación de productos terminados con un grupo de adolescentes y jóvenes y eso sirvió para saber su grado de aceptación.

Por tal motivo el objetivo de esta investigación es conocer el grado de aceptación de un snack saludable elaborado con nopal que no únicamente beneficie a los consumidores sino al medioambiente y a los productores del campo.

\section{MARCO TEÓRICO}

\subsection{La desforestación del planeta}

En los últimos 25 años se ha desforestado en el mundo una superficie boscosa equivalente en tamaño a la India (FAO, 2020) y México está considerado entre los países de mayor deforestación del planeta (GreenPeace, 2021) 
algo sumamente preocupante ya que no todo es de forma accidental como es el caso de los incendios, otras acciones nocivas que se realizan es por medio de la tala inmoderada de árboles, ya que la producción maderable deja grandes ganancias y desafortunadamente muy pocos productores vuelven a invertir en el cultivo forestal ya que es un recurso económico que en muy largo plazo se pueden explotar especies maderables (De Camino, 1997).

Otra práctica es el aspecto ganadero, se destruyen muchas áreas productivas para establecimiento de ganado, lo cual es una forma de obtener recursos en un plazo mucho más corto que la explotación de la madera por lo que es mejor negocio (Arbeletche, 2020), ya que para obtener algunas especies maderables el árbol tarda hasta 20 años en tener la madera en estado óptimo (Tapias-Padilla, 2015).

Pero el problema más lamentable y lo vemos en la época de sequía son los incendios forestales, que son un daño irreversible al que se somete nuestra flora y fauna, acabando el fuego con extensiones muy amplias de diversos tipos de vegetación generando a mediano plazo la erosión la cual consiste en la remoción de la capa superficial del suelo la cual es el sustento de la vegetación y con esto la degradación de las tierras de cultivo (Rivera-Ortíz, y otros, 2014), lo alarmante es que en el mundo cada cinco segundos se erosiona la superficie equivalente a un campo de futbol (FAO, 2021), superficie en la que en un futuro no podrá cultivarse nada y cada año tendremos una menor superficie para producir alimentos. Cuando se pierde la vegetación se tiene que cultivar en corto plazo las mismas especies u otras para evitar la erosión y por los costos desafortunadamente esta práctica en muchas ocasiones no se hace.

\subsection{El cultivo del nopal}

Una alternativa económica y de fácil acción es el poder cultivar esas tierras que están a punto de erosionarse con esta planta, ya que el nopal tiene características que pudieran ser muy útiles, como es su resistencia a las sequías (Palma, 2016), cada vez más frecuentes, así como su raíz que puede retener la capa de tierra (Thomé-Ortíz, Renard-Hubert, NavaBernal, \& de Souza-Valentino, 2014) y que llega a medir hasta 10 metros de longitud (Rodríguez-Mejía \& Macias Orozco, 1987), una variedad muy adaptable y de buen rendimiento es la Opuntia ficus-indica Mill (FAO, 2018), que es la usada en este trabajo.

El cultivo del nopal es muy sencillo, ya que, de una planta adulta y sana, de sus pencas centrales se cortan las pencas adultas exactamente en el cuello donde se une una penca con otra, se deja que cicatrice el corte y se siembra orientada la penca hacia la salida y puesta del sol introduciendo en la tierra un poco más de la mitad de cada penca (Rodríguez, 2010).

Dentro de las bondades de este proyecto es el mejorar el factor socioeconómico de los productores de nopal (Rodríguez-Mejía \& Macias Orozco, 1987) al buscar con ellos una relación ganar-ganar adquiriendo la materia prima directamente de los productores y con esto mejorando sus ingresos. Por lo que los productores tendrían tres alternativas con esta planta: la venta de sus frutos (tuna), sus pencas tiernas utilizarlas en este proyecto y las pencas de mayor edad que sirvieron para producir fruto se les puede proporcionar al ganado, como son: bovinos, ovinos, caprinos y porcinos (De La Rosa-Hidalgo, 2013).

Otro factor importante con el que cuenta el nopal es su riqueza en calcio y en fibra tanto insoluble como soluble (Bautista-Justo, y otros, 2010), por lo que un artículo que contenga nopal se puede considerar que aporta elementos benéficos para los futuros consumidores buscando con esto no ofrecer un producto alto en carbohidratos como muchas 
empresas lo hacen, que incrementa el sobrepeso en los niños y jóvenes, sino una alternativa que se pueda consumir con toda tranquilidad al ser de pocas calorías y rico en fibra dietética para una buena digestión y componente funcional para la alimentación del ser humano (Pérez-Valdez, 2012), incluso por sus mismas propiedades se le ha considerado auxiliar en el cuidado de la diabetes (Rodríguez-Riestra, 1985).

\subsection{Un producto con beneficios para la salud}

Al considerar el nopal un auxiliar en el cuidado de la diabetes y proponiendo que el producto sea orientado para adolescentes y jóvenes, ya que en México uno de cada tres adolescentes tiene sobrepeso u obesidad (Fernández-Cantón, Montoya-Núñez, \& Viguri-Uribe, 2011) y este es uno de los factores que desencadenan la diabetes (Escobedo-de la Peña, y otros, 2011), como el caso de la diabetes tipo 2 que anteriormente se nombraba diabetes de los adultos, desafortunadamente en la actualidad ya también es común en niños y jóvenes (MEDILINEPLUS, 2021). Donde también se encuentra en crecimiento alarmante en sólo diez años, casi al doble en México, es la diabetes tipo 1 pasando de 3.4 a 6.2 por cada 100 mil menores de 19 años (Prevention, 2021).

Por tal motivo la orientación del producto desarrollado es para las personas jóvenes, y para facilitar su consumo se hace de una forma habitual entre ellos que es el snack, lo cual da como consecuencia su facilidad de comer y transportar (Martínez-Cortés \& Romo-Herrera, 2018), así como muy versátil su utilización como es en reuniones, cines, trabajando, caminando, etc. (Juárez, 2020) y al estar familiarizados con esta presentación quizá resulta más fácil su aceptación, ya que algo alarmante que existe es que el $29 \%$ de los niños y jóvenes no consumen ninguna verdura (Prensa-UVM, 2015).

Vencer el estereotipo que tienen los snacks de no ser saludables (Hidalgo, 2011) es un reto de este trabajo, que tengan una alternativa dentro de sus mismos hábitos, pero que tiene la característica de no ser dañino, al contrario, el tener elementos que pueden beneficiar su salud.

\subsection{El proceso de encurtido y deshidratado del nopal}

El presente trabajo busca el desarrollo de un producto novedoso, de fácil fabricación y que no requiera de mucha inversión, por lo que se optó por un snack a base de tiras de nopal los cuales llevan a cabo dos procesos: el encurtido para que tenga un ligero sabor acido combinado con lo picoso que agrada al paladar de los jóvenes sobre todo los que tienen sobrepeso que es la condición del $74.7 \%$ de los que informaron el gusto por lo picoso (San Mauro-Martín, Mendive-Dubourdieu, Paredes-Barato, \& Garicano-Vilar, 2012)

Para la conservación del producto, como siguiente paso se lleva a cabo una deshidratación, es decir, que el producto pierda la mayor cantidad de agua para evitar su descomposición

Cabe aclarar que el encurtido es un método de conservación, pero en este caso sólo lo usaremos para mejorar el sabor del producto. Como ya se mencionó se utilizan las pencas tiernas y se cortan a lo largo.

Como se muestra en la figura número uno, se le prepara una mezcla de: sal (cloruro de sodio) el $49 \%$, bicarbonato de sodio como estabilizador de la clorofila (Almada, Cáceres, Machaín-Singer, \& Pulfer, 2005) el 49\% y ácido cítrico $2 \%$. Estos tres polvos se mezclan y posteriormente se incorporan a los nopales, esta actividad se hace con el fin de reducir la 
viscosidad que tiene el líquido que brota del nopal, lo que vulgarmente llaman baba de nopal. Después de dos horas se lavan los trozos de nopal para retirar la mezcla.

Figura 1. Proceso para reducir la viscosidad que tiene el líquido que brota del nopal

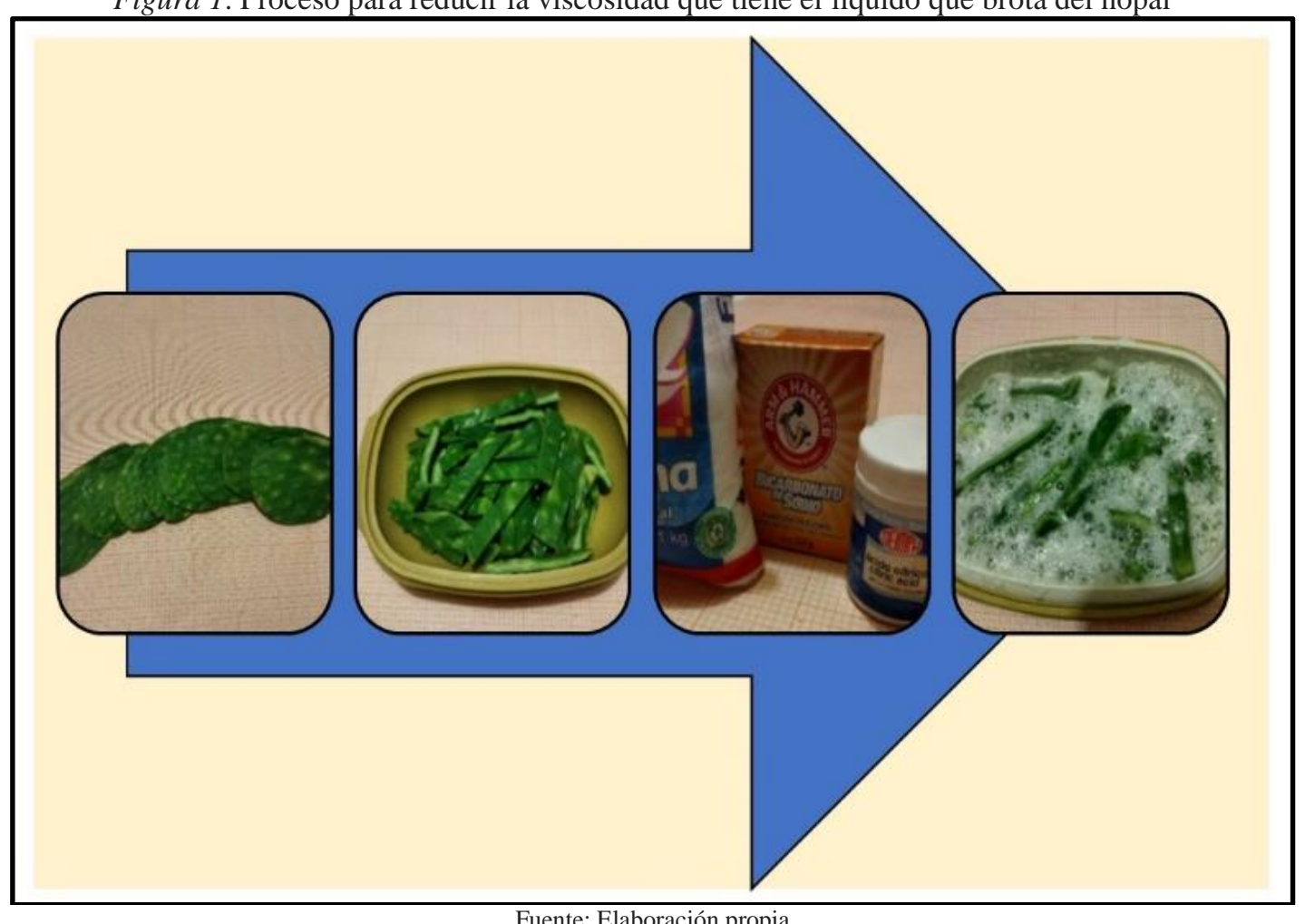

Fuente: Elaboración propia

Posteriormente para mejorar el sabor del nopal se coloca en un recipiente con un $30 \%$ de chiles en relación con los nopales, es decir, si coloco 100 gramos de nopales en trozo agrego 30 gramos de chile jalapeño.

Ambos, chiles y nopales se colocan en una sustancia preparada con vinagre de manzana $50 \%$, agua $44 \%$, sal $3 \%$, azúcar $1 \%$ $\mathrm{y}$ especies en forma equitativa (pimienta gorda, orégano, ajos y mejorana) un $2 \%$. Si se tiene un potenciómetro (para medir el $\mathrm{pH}$ ), este debe estar debajo de 4 (Clayton, Bush, \& Keener). Esto para estandarizar el proceso, es decir, que siempre se tenga la misma concentración de ácido en la mezcla, en el caso que fuéramos a utilizar este método para conservar deberíamos constantemente monitorear la acidez ya que si sube este valor el producto puede ser atacado por microorganismos. Pero en este caso, como ya se mencionó es únicamente para tener siempre la misma concentración.

Después de 72 horas, tanto el vinagre como los chiles jalapeños le transfieren parte de su sabor a los nopales, los cuales adquieren un sabor distinto al que tienen de origen, los pasos de este proceso se ilustran en la figura número dos: 
Figura 2. Proceso de nopales en escabeche para que adquieran sabor ácido y picoso

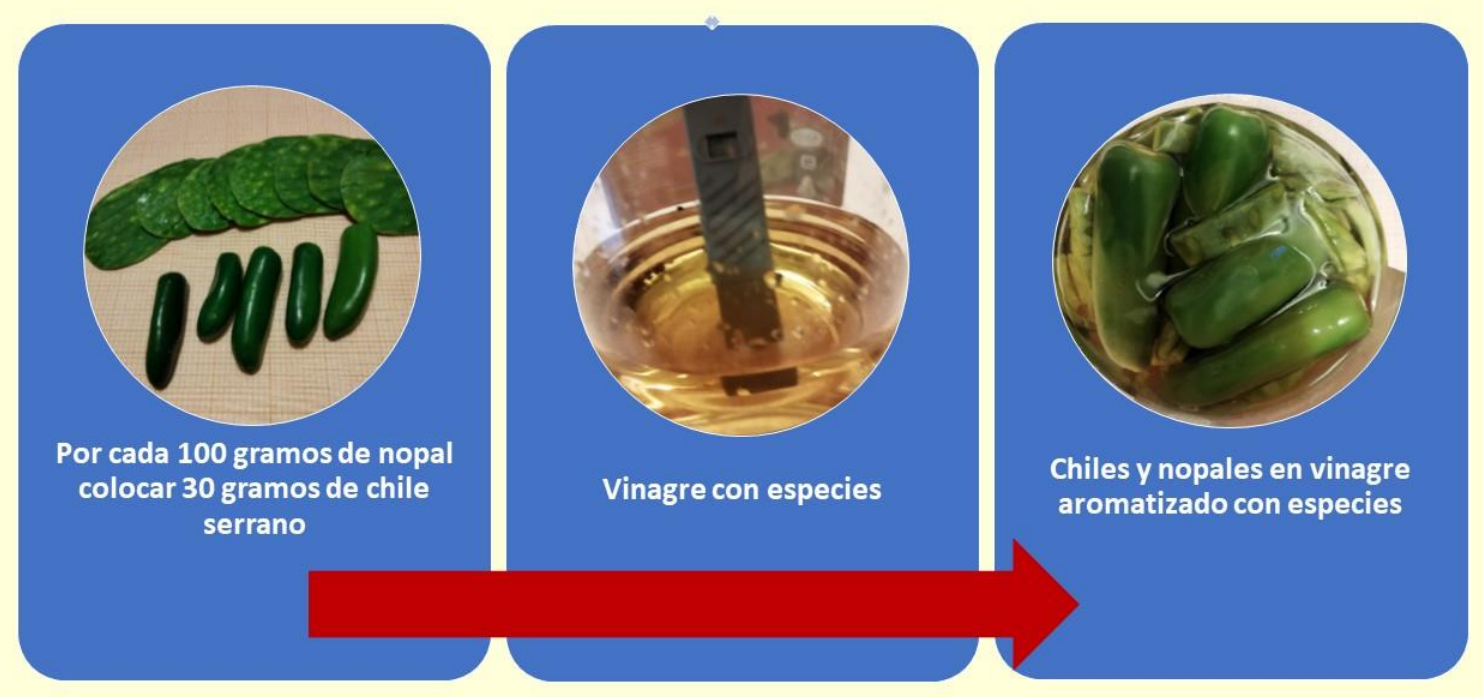

Fuente: Elaboración propia

El siguiente paso, para lograr su conservación, como ya se mencionó es el deshidratado, este método de conservación consiste en extraerle al producto la suficiente cantidad de agua para que los microorganismos no lo ataquen. Es como ocurre, por ejemplo, con el Bacalao Noruego, le ponen grandes cantidades de sal para que pierda agua y pierde tanta que se coloca a la intemperie y no se descompone con el paso del tiempo (Revista.UNAM.mx, 2005).

Por tal motivo se colocan las tiras de nopal en un deshidratador Hamilton Beach monofásico a una temperatura de 55 grados centígrados por cinco horas. Esta temperatura está dentro del rango que se sugiere para lograr la permeabilidad de las membranas de las frutas y hortalizas que oscila entre los 50 y 55 grados centígrados (Della-Roca, 2010).

En este proceso el producto pierde un $87.6 \%$ de agua, es decir, si yo coloco en el deshidratador 100 gramos de nopal a 55 grados centígrados por cinco horas al final voy a tener 12.4 gramos de nopal deshidratado.
Esta relación de temperatura $\left(55^{\circ} \mathrm{C}\right)$ y tiempo (5 horas) se obtuvo después de varias pruebas, en algunas era demasiada la temperatura y tiempo y el producto se carbonizaba. En otras pruebas era muy poco el tiempo y la temperatura y el producto en pocos días comenzaba a contaminarse, es decir, todavía tenía mucha agua y los microrganismos se desarrollaban. Por lo que el tratamiento ideal donde en su almacenaje posterior no se desarrollaron microorganismos patógenos fue el anteriormente mencionado.

Algo que resulta común es el cambio de color y textura del alimento con la deshidratación (Maupoey, Andrés-Grau, Barat Baviera, \& Albors-Sorolla, 2020) ya que al perder el $87.6 \%$ de su peso su tamaño se reduce de forma significativa, esto puede afectar la percepción visual del producto. En la figura número tres se muestra el producto antes y después de la deshidratación, donde es notoria la pérdida de tamaño, se presenta también su embolsado y como se presentó a los participantes 
Figura 2. Vista antes y después del deshidratado, embolsado y presentación para la degustación

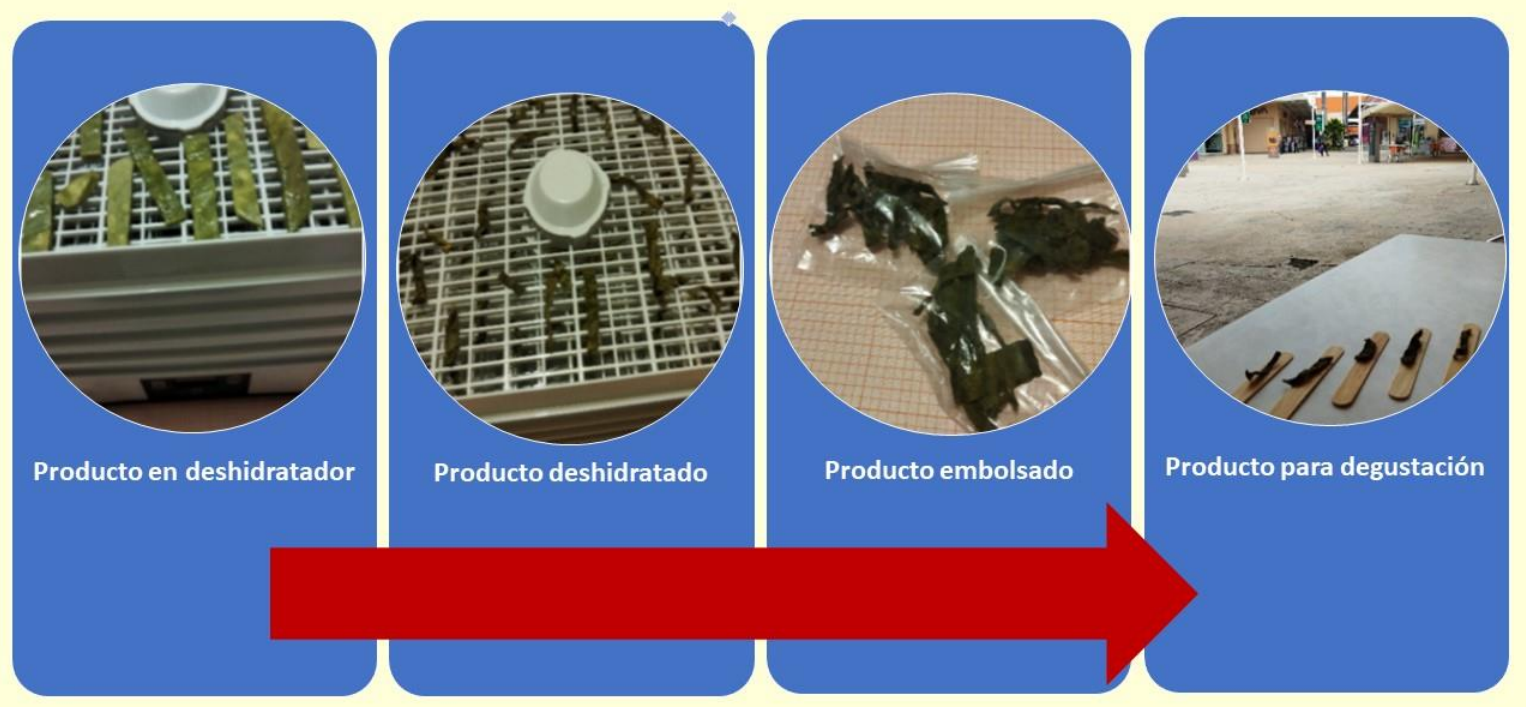

Fuente: Elaboración propia

El producto se empaca en bolsas de nueve por siete centímetros con un peso cada bolsa de 10 gramos.

\subsection{Análisis Sensorial de Alimentos}

Esto consiste en examinar las propiedades organolépticas del alimento por medio de los sentidos involucrados, en este caso van a ser los sentidos de la vista, tacto (textura en la boca al masticarlo) y gusto (sabor).

Es importante que los sentidos no se perciben todos en un momento, ya que los primeros en tener aparición son la vista y en su caso el olfato. Posteriormente tacto (textura), sabor y si el alimento es crujiente: el sonido.

\section{MÉTODO}

Como siguiente paso es importante obtener información requerida (IGAPE, 2006), en nuestro caso es sobre posibles interesados en el producto, conocer sus características predominantes (García-Córdoba, 2012) respecto al consumo de snacks sin que previamente visualicen el producto, por lo que el tipo de estudio correlacional incluyendo elementos descriptivos (Hernández-Sampieri, Fernández-Collado, \& Baptista-Lucio, 2014).

En la época actual resulta muy complicado realizar una degustación del producto por medio de un panel, ya que la pandemia del COVID 19 podría poner en riesgo a las personas invitadas, por tal motivo se realizó un panel ambulante en una plaza comercial en la ciudad de Tulancingo llamada Plaza Patio, donde en primer término deseamos saber, entre los adolescentes y jóvenes el interés por consumir un snack saludable, posteriormente presentarles el producto para su degustación y saber si resulto de su agrado a la vista, su textura y sabor. En la siguiente etapa, utilizando como herramienta estadística la t de Student que nos es útil para conocer si difieren de manera significativa dos grupos (adolescentes y jóvenes) respecto al posible consumo del 
producto (Castilla-Serna \& Cravioto, 1991) también otra bondad de esta prueba es que se utiliza para muestras pequeñas, menos de 30 sujetos de estudio (Sánchez-Turcios, 2015), que por la pandemia es lo que se logró que participarán.

Los adolescentes y jóvenes fueron divididos por edades, es decir, algo parecido al manejo de la Organización Mundial de la
Salud, como adolescencia temprana y tardía, los lo que para este caso los vamos a agrupar en adolescentes de 12 a 14 años y jóvenes de 15 a 19 años (Secretaria de Salud, 2015). Los participantes fueron dieciséis adolescentes y dieciocho jóvenes a los que como inicio se les preguntó si les interesaría probar un snack con la característica de ser saludable obteniendo los siguientes resultados que se aprecian en la tabla número uno:

Tabla 1. Adolescentes y jóvenes que desearon participar en la degustación

\begin{tabular}{|c|c|c|c|}
\hline DIVISIÓN & SI & NO & TOTAL \\
\hline Adolescentes & 16 & 4 & 20 \\
\hline Jóvenes & 18 & 3 & 21 \\
\hline Total & 34 & 7 & 41 \\
\hline
\end{tabular}

Los adolescentes y jóvenes que desearon participar fueron en total 34, 16 adolescentes y 18 jóvenes, por lo que el estudio se centró en ellos.

\section{RESULTADOS:}

Se les proporcionó a los interesados en participar en la prueba el producto para que después de degustarlo respondieran por medio de la Escala de Lickert tres preguntas alusivas al producto con lo que respecta a las propiedades organolépticas (apariencia, textura y sabor), donde se miden cinco alternativas con diversos grados de aceptación (Monje-Álvarez, 2011), para este caso van desde el enunciado de Muy bien hasta Muy mal (UAH):

El primer caso fue la apariencia del producto, obteniendo los siguientes valores que aparecen en la tabla número dos, tanto en los adolescentes como en los jóvenes:

Tabla 2. Valoración del aspecto del producto

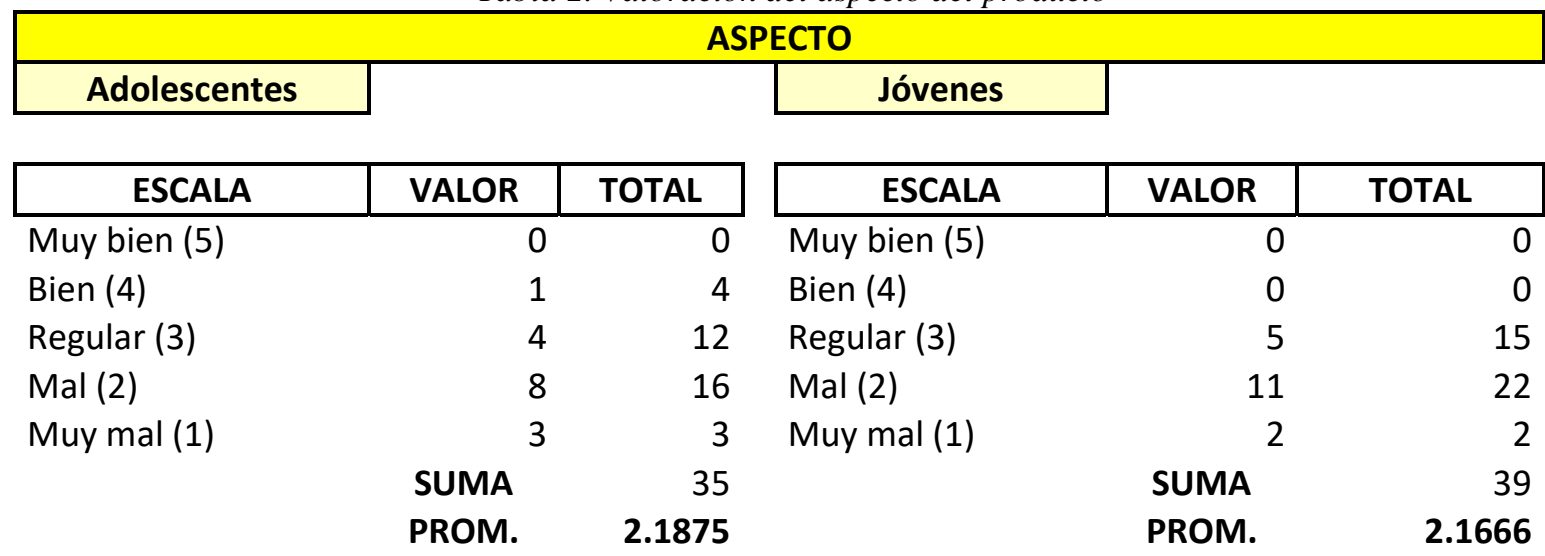

Fuente: Elaboración Propia 
Los valores obtenidos en Escala de Lickert fueron 2.18 y 2.16 de los adolescentes y los jóvenes respectivamente.
Posteriormente valoraron los participantes la textura, ambos grupos (adolescentes y jóvenes) los resultados que se obtuvieron son los de la tabla número tres:

Tabla 3. Valoración de la textura del producto

\begin{tabular}{|c|c|c|c|c|c|}
\hline \multicolumn{6}{|c|}{ TEXTURA } \\
\hline Adolescentes & & & Jóvenes & & \\
\hline ESCALA & VALOR & TOTAL & ESCALA & VALOR & TOTAL \\
\hline Muy bien (5) & \multicolumn{2}{|r|}{10} & Muy bien (5) & \multicolumn{2}{|r|}{15} \\
\hline Bien (4) & \multicolumn{2}{|r|}{12} & Bien (4) & \multicolumn{2}{|c|}{3} \\
\hline Regular (3) & \multicolumn{2}{|r|}{24} & Regular (3) & \multicolumn{2}{|c|}{7} \\
\hline Mal (2) & \multirow{2}{*}{\multicolumn{2}{|c|}{$\begin{array}{l}2 \\
1\end{array}$}} & Mal (2) & \multicolumn{2}{|c|}{4} \\
\hline \multirow[t]{3}{*}{ Muy mal (1) } & & & Muy mal (1) & \multicolumn{2}{|c|}{1} \\
\hline & SUMA & 51 & & SUMA & 57 \\
\hline & PROM. & 3.1875 & & PROM. & 3.1666 \\
\hline
\end{tabular}

Fuente: Elaboración Propia

La textura tuvo el valor de: 3.1875 para los adolescentes y 3.1666 para los jóvenes.
Por último, se valoró los resultados que se le asignaron al sabor, los cuales fueron plasmados en la tabla número cuatro:

Tabla 4. Valoración del sabor del producto

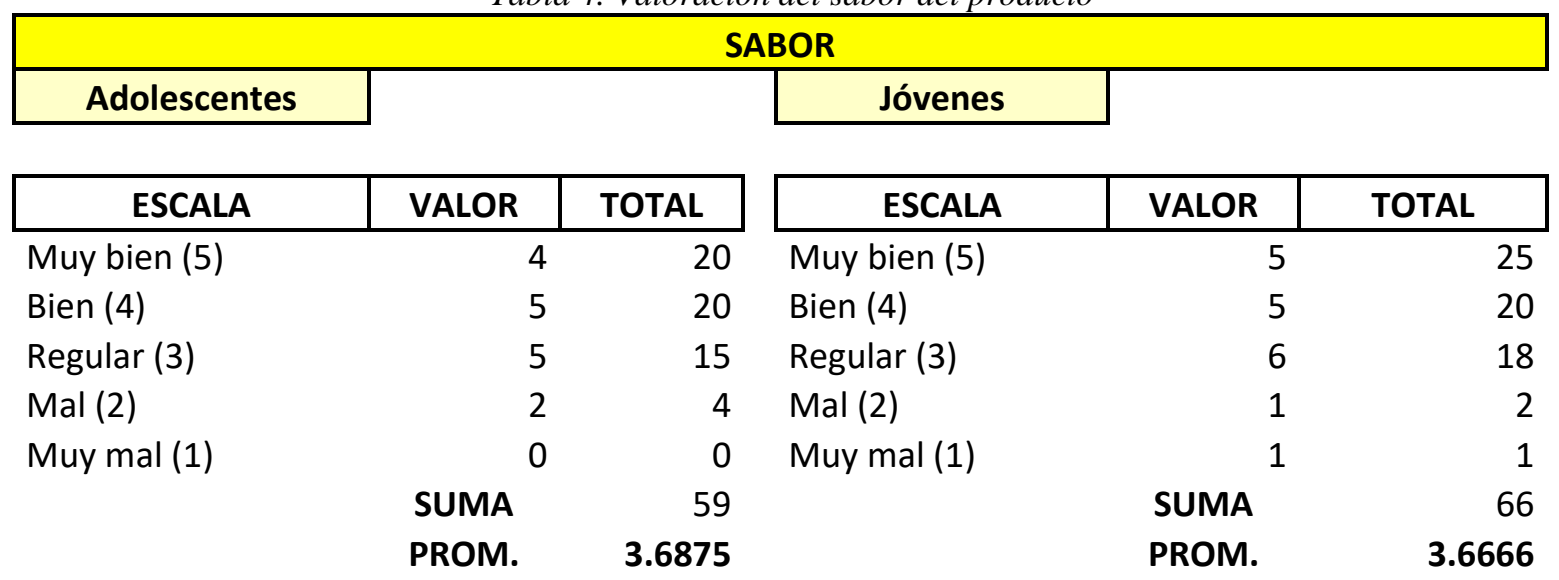

Fuente: Elaboración Propia

Los valores en promedio que se obtuvieron para adolescentes y jóvenes fueron 3.6875 y 3.6666 respectivamente.
Para saber si ambos grupos tienen el mismo comportamiento y así enfocarnos de manera uniforme respecto a la aceptación o no, 
vamos a aplicar la prueba de t de Student y saber si el tratamiento va a ser similar y ya no dividirlos, bajo la siguiente hipótesis alternativa, en la cual hemos aglutinado las tres pruebas realizadas del mismo número de sentidos: ver, tocar (textura) y gustar como las propiedades organolépticas:

- $\mathbf{H}_{\mathbf{A}}=$ Los adolescentes tuvieron la misma aceptación del snack respecto a las propiedades organolépticas que los jóvenes.

Por lo que la hipótesis nula sería:

- $\mathbf{H}_{\mathbf{0}}=$ Los adolescentes no tuvieron la misma aceptación del snack respecto a las propiedades organolépticas que los jóvenes.

Como siguiente paso se colocan los resultados de las tres pruebas en una tabla de Excel ${ }^{\circledR}$ y se obtiene la t de Student, esto se puede apreciar en la tabla número cinco:

Tabla 5. Valores obtenidos de la Prueba de t de Student

\begin{tabular}{|c|c|c|c|c|}
\hline \multirow{2}{*}{\begin{tabular}{r|} 
Adolescent. \\
0
\end{tabular}} & \multirow{2}{*}{$\begin{array}{r}\text { Jovenes } \\
0\end{array}$} & \multicolumn{3}{|c|}{ t-Test: Paired Two Sample for Means } \\
\hline & & & & \\
\hline 1 & 0 & \multicolumn{3}{|c|}{ Variable 1 Variable 2} \\
\hline 4 & 5 & Mean & 3.2 & 3.6 \\
\hline 8 & 11 & Variance & 6.314286 & 9.114286 \\
\hline 3 & 2 & Observations & 15 & 15 \\
\hline 2 & 3 & Pearson Correlation & 0.924613 & \\
\hline 3 & 3 & \multicolumn{2}{|l|}{ Hypothesized Mean Diff $\epsilon$} & \\
\hline 8 & 7 & df & 14 & \\
\hline 2 & 4 & t Stat & -1.30931 & \\
\hline 1 & 1 & $\mathrm{P}(\mathrm{T}<=\mathrm{t})$ one-tail & 0.105756 & \\
\hline 4 & 5 & t Critical one-tail & 1.76131 & \\
\hline 5 & 5 & $\mathrm{P}(\mathrm{T}<=\mathrm{t})$ two-tail & 0.211512 & \\
\hline 5 & 6 & t Critical two-tail & 2.144787 & \\
\hline 2 & 1 & & & \\
\hline 0 & 1 & & & \\
\hline
\end{tabular}

Fuente: Elaboración Propia

La cual tiene un valor de 2.1447, para este caso utilizamos alfa con un valor de 0.05 (Anderson, Sweeney, \& Williams, 2008), lo que indica que la probabilidad de observar las diferencias en los datos al azar es de solo el $5 \%$.
Consultamos el valor en tablas de $\mathrm{t}$ de Student que aparecen en la tabla número seis, donde en la columna se tienen las personas que participaron que fueron $34 \mathrm{y}$ en la fila el valor de alfa que es de 0.05 , con ambos valores obtuvimos lo siguiente: 
Tabla 6. Valores de la Prueba de t de Student

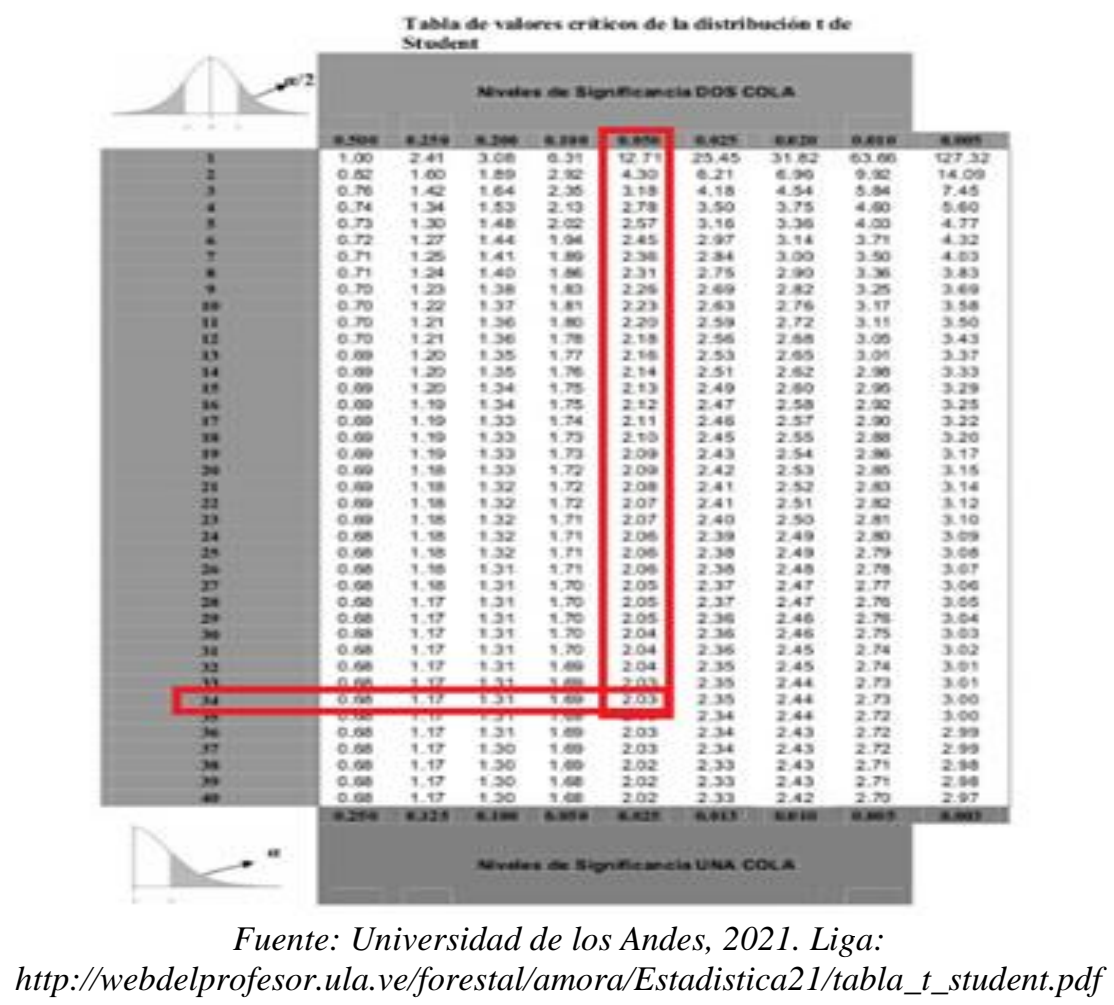

En las tablas el valor obtenido es de 2.03 el cual es menor que el valor de la Prueba de $\mathrm{t}$ de Student calculado que fue de 2.14, por lo que se cumple la hipótesis alternativa que es: Los adolescentes tuvieron la misma aceptación del snack respecto a las propiedades organolépticas que los jóvenes.

\section{CONCLUSIONES}

El 20\% de los adolescentes y el $14.28 \%$ de los jóvenes no mostraron interés por el producto, es decir, un snack saludable no es algo que les agrade incluir en su consumo, esto fue incluso antes de ver el producto y degustarlo. Aunque también su respuesta se pudo deber a que sentían que el participar podía poner en riesgo su salud al ser contagiados por Coronavirus.

Con lo que respecta a la apariencia del producto fue algo que no resultó agradable al grupo de adolescentes y jóvenes que participaron, esto pone en riesgo la viabilidad del proyecto ya que de entrada el primer contacto que tiene el consumidor en potencia es el visual, los valores fueron los más bajos de las tres preguntas 2.16 y 2.18 .

Respecto a la textura del mismo al momento de tenerlo en su boca tuvo un valor neutro, es decir, la tendencia del valor de la Escala de Lickert se ubicó cercano al tres: 3.16 y 3.18 .

El sabor fue la prueba que obtuvo mayor puntuación con los valores de 3.66 y 3.68 , esto quiere decir que si se logró el transmitir el sabor deseado y fue agradable a los participantes. Esto de alguna forma es la fortaleza del producto desarrollado y lo que al final se desea, pero no lo es todo ya que antes de llevarlo a su boca existe el sentido de la vista.

Por lo anteriormente expuesto la apariencia del producto es básico, por buen sabor que se tenga si no resulta agradable a la 
vista el producto a desarrollar se complica su aceptación.

El último dato que se buscó es saber si el grado de aceptación de los adolescentes y los jóvenes fue similar, esto con el objeto de evitar en un futuro el trabajar por separado ambos grupos, es decir, las mejoras que se le realicen en un futuro al producto no tiene caso hacer los dos grupos.

Es importante que el consumidor tenga la alternativa de probar el producto, el cual tiene muchas posibilidades de resultar de su agrado, aunque tiene una barrera que es su apariencia, lo que ocurre que al perder el $87.6 \%$ por medio de aire caliente se reduce su tamaño y no resulta muy agradable a la vista, por lo que para continuar y mejorar con este mismo producto se tienen dos sugerencias:

1. Llevar a cabo la perdida de agua no por medio de un deshidratador, sino por medio de la liofilización, la cual consiste en un proceso más suave para extraer el agua a los productos y es el mejor método para secar compuestos orgánicos o inorgánicos sin alterar su composición cualitativa o cuantitativa (Grupo GIDOLQUIM, 2014), es decir, el producto queda casi de las mismas dimensiones, pero un poco poroso por el agua que pierde, el problema que esta técnica es más costosa.

2. El incluir en otros snacks de frutas deshidratadas algunos trozos de este nopal, así la apariencia se puede enmascarar al estar revuelto con otros productos similares.
3. Se puede pulverizar el producto y combinarse con otros productos en polvo como el chile con limón, este sería otro trabajo de investigación.

Consideramos importante continuar con el seguimiento del proyecto ya que es una verdura que pueden consumir los adolescentes y jóvenes, ya que como lo vimos tres de cada diez niños y jóvenes no consumen verduras y que uno de cada tres adolescentes y jóvenes tienen sobrepeso u obesidad, por lo que tienen la tendencia a desarrollar diabetes en un futuro y el nopal puede ser un auxiliar en este problema de salud.

Otro aspecto que no debemos perder de vista es que el fomentar el cultivo del nopal coadyuva para evitar la erosión, así mientras más se cultive mejores tierras vamos a tener y vamos a ofrecerles fuentes de trabajo a nuestros campesinos por medio de un proyecto donde ellos producen la materia prima.

El próximo desarrollo de un producto que mejore su apariencia es importante ya no separar a las personas que participan entre adolescentes y jóvenes, ya que su comportamiento fue similar, eso lo obtuvimos mediante la prueba de $t$ de Student.

Finalmente es importante considerar realizar nuevamente la degustación bajo otras condiciones: sin pandemia, ya que el peligro de adquirir el COVID hizo que muchos participantes no desearan participar o participar predispuestos a un contagio y esto pudo haber generado un sesgo en la información recabada. 


\section{REFERENCIAS}

Almada, M., Cáceres, M., Machaín-Singer, M., \& Pulfer, J. (2005). Guia de uso de secadores solares. Asunción, Paraguay: UNESCO-Fundación Celestina Perez de Almada.

Anderson, D., Sweeney, D., \& Williams, T. (2008). Estadística para Administración y Economía. México: CENGAGE Learning.

Arbeletche, P. (Enero de 2020). El Agronegocio en Uruguay: su evolución y estrategias cambiantes en el Siglo XXI. RIVAR, 7 (19), pág. 120. doi:10.35588/rivar.v7i19.4355

Bautista-Justo, M., Pineda-Torres, R., Camarena-Aguilar, E., Alanís-Guzman, G., De Mota, V., \& Barbosa-Corona, J. (septiembre-diciembre de 2010). El nopal fresco como fuente de fibra y calcio en panqués. Acta Universitaria, 11-12. Recuperado el enero 25 de 2021, de https://www.redalyc.org/articulo.oa?id=41615947002

Castilla-Serna, L., \& Cravioto, J. (1991). Estadística Simplificada: para la investigación de ciencias de la salud. México: Trillas.

Clayton, K., Bush, D., \& Keener, K. (s.f.). Metodos para la conservación de alimentos. Lafayette: Purdue University. Recuperado el 15 de enero de 2021, de https://www.extension.purdue.edu/extmedia/FS/FS-15-S-W.pdf

De Camino, R. (1997). "Son las plantaciones forestales un negocio rentable". San José, Costa Rica: Actas Tercer Congreso Forestal Nacional. Obtenido de https://www.researchgate.net/profile/Ronnie-De-

Camino/publication/255820573_Son_las_Plantaciones_Forestales_un_Negocio_Rentable/lin ks/54e27d150cf2966637969e05/Son-las-Plantaciones-Forestales-un-Negocio-Rentable.pdf

De La Rosa-Hidalgo, E. (2013). Evaluación bromatológica y digestibilidad in vitro de dietas de nopal (Opuntía strepthacanta) e inflorececias de maguey. (U. A. Narro, Ed.) Saltillo, Coahuila, México: División de Ciencia Animal.

Della-Roca, P. (2010). Secado de alimentos por métodos combinados: Deshidratación Osmótica y secado por microondas y aire caliente. (F. R. Aires, Ed.) Buenos Aires, Argentina: Universidad Tecnológica Nacional. Obtenido de https://docplayer.es/7266922-Secado-dealimentos-por-metodos-combinados-deshidratacion-osmotica-y-secado-por-microondas-yaire-caliente.html

Escobedo-de la Peña, J., Buitrón-Granados, L., Ramírez-Martínez, J., Chavira-Mejía, R., Schargrodsky, H., \& Marcet, C. (2011). Diabetes en México: Estudio CARMELA. Cirugía y Cirujanos, 79 (5), 424. Obtenido de https://www.medigraphic.com/cgi$\mathrm{bin} /$ new/resumen.cgi?IDARTICULO=31342

FAO. (2018). Ecología del cultivo, manejos y usos del nopal. Centro Internacional de Investigaciones Agrícolas en Zonas Áridas. Roma: Universitá Degli Studi di Palermo . Recuperado el 22 de febrero de 2021, de http://www.fao.org/3/i7628es/I7628ES.pdf

FAO. (2020). Políticas Pecuarias. Ganadería y Deforestación. Santiago de Chile: Subdirección de Información Ganadera y de Análisis y Política del Sector. Dirección de Producción y Sanidad Animal. Obtenido de http://www.fao.org/3/a0262s/a0262s.pdf

FAO. (11 de febrero de 2021). Detengamos la erosión del suelo para garantizar la seguridad alimentaria en el futuro. (FAO, Editor, \& D. 1. futuro, Productor) Obtenido de Organización de las Naciones Unidas para la Alimentación y la Agricultura: http://www.fao.org/faostories/article/es/c/1193735/

Fernández-Cantón, S., Montoya-Núñez, Y., \& Viguri-Uribe, R. (2011). Sobrepeso y obsesidad en menores de 20 años de edad en México. Boletín Médico del Hospital Infantil de México, 68 (1), 80. Obtenido de http://www.scielo.org.mx/scielo.php?script=sci_arttext\&pid=S166511462011000100011

García-Córdoba, F. (2012). El Cuestionario. Ciudad de México: LIMUSA.

GreenPeace. (22 de abril de 2021). 5 Datos para reflexionar el día de la Tierra. Obtenido de Green Peace México: https://www.greenpeace.org/mexico/blog/4390/5-datos-para-reflexionar-enel-dia-de-la-tierra/ 
Grupo GIDOLQUIM. (4 de abril de 2014). Tema 4. El proceso de liofilización. (U. d. Barcelona, Editor) Recuperado el 10 de marzo de 2021, de Técnicas y operaciones avanzadas en el laboratorio químico: http://www.ub.edu/talq/es/node/261

Hernández-Sampieri, R., Fernández-Collado, C., \& Baptista-Lucio, P. (2014). Metodología de la investigación. Ciudad de México: Mc Graw Hill Education.

Hidalgo, M. A. (2011). Consumo de comida rápida, snacks y gaseosas en adolescentes obesos y normales de instituciones educativas estatales de San Isidro. (U. N. Marcos, Ed.) Lima, Perú: Facultad de Medicina Humana. Obtenido de https://www.researchgate.net/publication/204113629_Consumo_de_comida_rapida_snacks_ y_gaseosas_en_adolescentes_obesos_y_normales_de_instituciones_educativas_estatales_de_ San_Isidro? enrichId=rgreq-fb211 adf3338ea017669e08dbcfb2f34XXX\&enrichSource $=$ Y292ZXJQYW

IGAPE. (2006). Manuales Prácticos - Como realizar un estudio de mercado. Galicia: BIC.GaliciaFSE.

Juárez, C. (7 de mayo de 2020). Snacks, segmento que va por más. Obtenido de The Food Tech: https://thefoodtech.com/tendencias-de-consumo/snacks-segmento-que-va-por-mas/

Martínez-Cortés, A. C., \& Romo-Herrera, M. (2018). Desarrollo de una formulación para un snack nutritivo a base de yogur. (U. N. México, Ed.) Cuautitlán Izcall, Estado de México, México: Facultad de Estudios Superiores Cuautitlán. Obtenido de http://132.248.9.195/ptd2018/marzo/0771780/Index.html

Maupoey, P., Andrés-Grau, A., Barat Baviera, J., \& Albors-Sorolla, A. (2020). Introducción al secado de alimentos por aire caliente. Valencia, España: Universitat Politecnica de Valencia.

MEDILINEPLUS. (23 de febrero de 2021). Diabetes en niños y adolescentes. (Biblioteca Nacional de Medicina de los Estados Unidos) Obtenido de MediLinePlus - Información de salud: https://medlineplus.gov/spanish/diabetesinchildrenandteens.html\#: :text=Hoy\%20en\%20d\% $\mathrm{C} 3 \% \mathrm{ADa} \% 20 \mathrm{~m} \% \mathrm{C} 3 \% \mathrm{~A} 1 \mathrm{~s} \% 20$ personas,no\%20usa\%201a\%20insulina\%20adecuadamente.

Monje-Álvarez, C. A. (2011). Metodología de la Investigación Cuantitativa y cualitativa: Guía didáctica. (U. Surcolombiana, Ed.) Neiva: Facultad de Ciencias Sociales y Humanas.

Palma, F. (2016). Academicas investigan propiedades del nopal para instalarlo en la dieta de los chilenos. Santiago: Universidad de Chile. Obtenido de https://www.uchile.cl/noticias/124819/academicas-investigan-propiedades-del-nopal-parainstalarlo-en-dieta

Pérez-Valdez, E. (2012). Estudio In vitro e in vivo del proceso digestivo de fibra dietética y almidón resistente. Morelia, Michoacán: Universidad Michoacana de San Nicolás de Hidalgo. Obtenido de http://www.remeri.org.mx/portal/REMERI.jsp?id=oai:bibliotecavirtual.dgb.umich.mx:12345 $6789 / 4860$

Prensa-UVM, S. d. (18 de noviembre de 2015). 91\% de los niños y jóvenes en México consume más de 5 porciones de bebidas azucaradas al día. Obtenido de Universidad del Valle de México: https://laureate-comunicacion.com/prensa/91-de-los-ninos-y-jovenes-en-mexico-consumemas-de-5-porciones-de-bebidas-azucaradas-al-dia/\#.YIisAbVKjIU

Prevention, C. f. (27 de febrero de 2021). Estadísticas sobre diabetes tipo 1. Obtenido de BeyondType: https://es.beyondtype1.org/estadisticas-sobredt1/\#: :text=entre\%20los\%20ni\%C3\%B1os.,La\%20tasa\%20de\%20incidencia\%20de\%20diabetes\%20Tipo\%201\%20en\%20ni\%C3\%B1o s,diagnostica\%201a\%20diabetes\%20Tipo\%201.

Revista.UNAM.mx. (10 de septiembre de 2005). Métodos de conservación de alimentos marinos. Revista Digital Universitaria, 6 (9), 1. Recuperado el 4 de mayo de 2021, de http://www.revista.unam.mx/vol.6/num9/art90/art90-3.htm/

Rivera-Ortíz, P., Rivera-Lárraga, J., Andrade-Limas, E., de la Garza-Requena, F., Castro-Meza, B., \& Delmonte-Serrato, F. (2014). Medición de la erosión en Cárcavas por medio de imágenes 
de satélite. (S. M. Suelo, Ed.) Tierra Latinoamericana, 32 (1), 13. Obtenido de https://www.redalyc.org/articulo.oa?id=57330740002

Rodríguez, C. (2010). Establecimiento de una plantación de nopal verdura y algunas pruebas de deshidratación de nopalito. Revista Salud Pública y Nutrición, 5, 238. Obtenido de http://respyn2.uanl.mx/especiales/2010/ee-05-2010/documentos/19.pdf

Rodríguez-Mejía, J., \& Macias Orozco, A. (1987). Establecimiento de plantaciones de nopal (Opuntia SPP) para tuna en la región sureste de Zacatecas. (F. d. Agricultura, Ed.) Guadalajara, Jalisco, México: Universidad de Guadalajara.

Rodríguez-Riestra, C. (1985). El nopal una alternativa de producción para las "tierras flacas" del estado de Jalisco. (F. d. Agricultura, Ed.) Guadalajara, Jalisco, México: Universidad de Guadalajara.

San Mauro-Martín, I., Mendive-Dubourdieu, P., Paredes-Barato, V., \& Garicano-Vilar, E. (2012). Programación nutricional del gusto y la tolerancia al picante. Nutrición Hospitalaria, 33 (4), 927. Obtenido de https://scielo.isciii.es/pdf/nh/v33n4/24_original23.pdf

Sánchez-Turcios, R. A. (2015). T de Student: Usos y abusos. Revista Mexicana de Cardiología, 26 (1), 59. Obtenido de http://www.scielo.org.mx/pdf/rmc/v26n1/v26n1a9.pdf

Secretaria de Salud. (20 de agosto de 2015). ¿Qué es la adolescencia? Obtenido de Gobierno de la Republica: https://www.gob.mx/salud/articulos/que-es-laadolescencia\#: :text=La\%20Organizaci\%C3\%B3n\%20Mundial\%20de\%20la,de\%2015\%20a $\% 2019 \% 20 \mathrm{a} \% \mathrm{C} 3 \% \mathrm{~B} 1 \mathrm{os}$.

Tapias-Padilla, C. A. (2015). Plan de negocios para el desarrollo de un proyecto forestal-comercial de Teca en el Magdalena Medio. Facultad de Ingenierías Físico Mecanicas, Escuela de Estudios Industriales y Empresariales. Bucaramangara: Universidad Industrial de Santander. Obtenido de http://tangara.uis.edu.co/biblioweb/tesis/2015/156295.pdf

Thomé-Ortíz, H., Renard-Hubert, M., Nava-Bernal, E., \& de Souza-Valentino, A. (julio-septiembre de 2014). La Ruta del Nopal (Opuntia Spp.). Turismo Reestructuración Productiva en el Suelo Rural de la Ciudad de México. (U. d. Sul, Ed.) Rosa dos Ventos, 6 (3), 392. Obtenido de https://www.redalyc.org/articulo.oa?id=473547041006

UAH. (s.f.). Manual, normas y orientaciones para la elaboración del trabajo de grado. Caracas, Venezuela: Universidad de Alejandro Humboldt. 